\title{
ARTICLE
}

\section{Liquid-phase synthesis of butyl tert-butyl ether catalysed by ion- exchange resins: kinetic modelling through in-depth model discrimination}

Received 00th January 20xx, Accepted 00th January 20xx DOI: $10.1039 / x 0 x \times 00000 x$

\author{
Jordi Hug Badia, ${ }^{a}$ Carles Fité, ${ }^{\text {a }}$ Roger Bringué, ${ }^{a}$ Eliana Ramírez ${ }^{\text {a }}$ and Montserrat Iborra ${ }^{a}$
}

\section{Introduction}

Reaction between isobutene (IB) and 1-butanol (BuOH) produces butyl tert-butyl ether (BTBE) and can be catalysed by acidic ion-exchange resins. BTBE is an interesting alternative to methyl and ethyl tert-butyl ethers (MTBE and ETBE, respectively) to be used as oxygenated high-octane component in current gasoline formulations. Since 1-butanol can be obtained through fermentation of non-edible biomass, ${ }^{1,2}$ it would allow a reduction of the fossil fuel use and dependence in gasoline production. BTBE can be produced at industrial scale in the same reaction units than MTBE or ETBE, in contrast to the next generation biofuels (stemmed from lignocellulose, non-food materials, algal biomass, and energy crops grown on marginal lands) that these days are receiving more attention, but still being under development. ${ }^{3,4}$ Thus, BTBE is a feasible option to find cleaner alternatives to traditional automotive fuels in the short- and midterm.

In the course of the addition of IB to BuOH to form BTBE, two side reactions can take place simultaneously depending on the operating conditions (Figure 1): isobutene hydration to form

a. Chemical Engineering and Analytical Chemistry Department, Faculty of Chemistry, University of Barcelona, Martí i Franquès 1 11, 08028 Barcelona, Spain.

E-mail: fite@ub.edu

Electronic Supplementary Information (ESI) available. See DOI: $10.1039 / \times 0 \times x 00000 x$ tert-butyl alcohol (TBA), and isobutene dimerisation to form 2,4,4-trimethyl-1-pentene (TMP1) and 2,4,4-trimethyl-2pentene (TMP2). Few literature references focused on BTBE synthesis can be found. The most significant works are devoted to the reaction thermodynamics and to the reactivity of primary alcohols with isobutene. ${ }^{5-9}$ No data have been found concerning the BTBE etherification kinetics, which is crucial for the potential production of BTBE in industrial units. Due to the reaction similarities, BTBE synthesis is expected to proceed through a mechanism similar to that of MTBE or ETBE and, therefore, reaction rates could be explained by means of

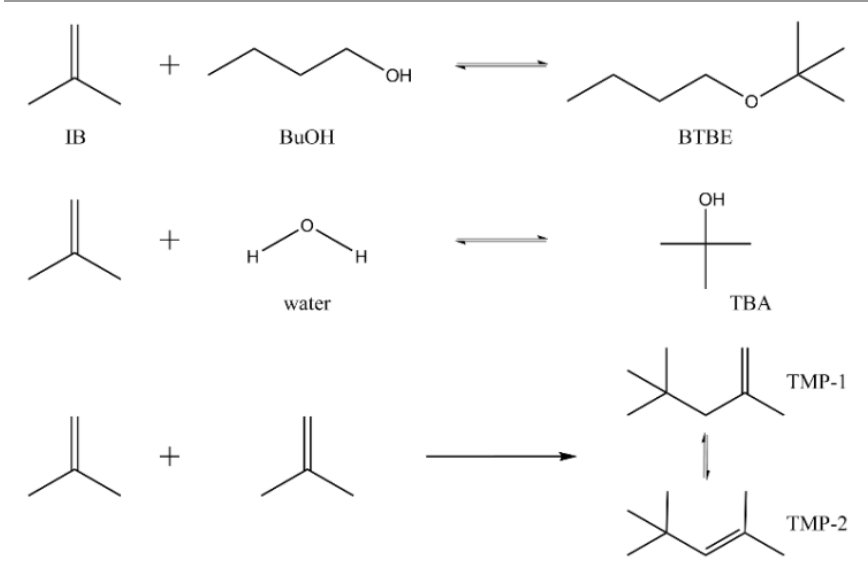


an analogous kinetic model.

MTBE and ETBE etherifications have been studied extensively throughout the years. ${ }^{10-16}$ More recently, the production of propyl tert-butyl ether (PTBE), the next ether in the analogous series, obtained by addition of 1-propanol to isobutene, has also been investigated. ${ }^{17}$ According to the literature, all these etherification reactions are reversible and exothermic, and the olefin-alcohol-ether mixtures behave strongly non-ideally. The reported reaction rate expressions are basically derived from Langmuir-Hinshelwood-Hougen-Watson (LHHW) or EleyRideal (ER) formalisms. It is expected that these reactions proceed through a similar mechanism. However, there are some discrepancies among the published works concerning mainly the number of active sites involved in the ratedetermining step and the compounds that actually adsorb significantly on the resin. Possible reasons of such discrepancies are to have tested a limited number of candidate models, to have chosen a model among others with a similar goodness of fit, to have included non significant effects in the kinetic equation, or to have excluded significant effects. The present in-depth kinetic model discrimination study is motivated to assure that all plausible models are considered in the discrimination procedure, and that all parameters included in the proposed kinetic equation are significant.

\section{Building the kinetic models}

The candidate models of the BTBE synthesis have been developed from the LHHW and ER formalisms, because it is a heterogeneously catalysed system. The form of a given kinetic equation is characterised by a set of compounds that adsorb on the catalyst, a set of elementary reactions, and the reaction step being the rate-determining step. Following the approach of our previous works, ${ }^{17,18}$ the search of the best kinetic model starts with the proposal of all kinetic equations to be tested. They have been obtained from the systematic combination of all possible rate-determining steps, adsorbed and nonadsorbed species on the catalyst, and significant or nonsignificant temperature dependence of every parameter, as well as the possible inclusion of a term accounting for the effect on rates of the interaction between the resin and the liquid mixture. The aim of this procedure is to avoid dismissing the true model (i.e., the one that most accurately describes the physicochemical reality of the etherification reaction) from the set of candidate models. The BTBE reacting mixture is assumed to be highly non-ideal, similarly to the analogous etherification systems. Therefore, activities were used instead of concentrations and estimated by means of the UNIFACDortmund method, which is an enhancement of the widely known UNIFAC method. ${ }^{19}$

All proposed equations match the same general expression (Equation 1). Models differ in the developed form of each term in the kinetic equation (Table 1 ).

$\left\{\begin{array}{c}\text { reaction } \\ \text { rate }\end{array}\right\}=\left\{\begin{array}{c}\text { kinetic } \\ \text { term }\end{array}\right\} \frac{\{\text { driving force }\}}{\{\text { adsorption term }\}^{n}}\left\{\begin{array}{c}\text { resin-medium } \\ \text { interaction }\end{array}\right\}$

As seen in Table 1, the kinetic term is $k^{\prime}$, an apparent kinetic coefficient, with the temperature dependence shown in Equation 2, since it consists of a product of the kinetic constant of the rate determining step (assumed to follow the

Table 1 Alternative forms of the kinetic equation terms (Equation 1). $a$ stands for activity; $b$ are binary-type variables (allowed values: 1 or 0 ), used to show generalised equation expressions; subscripts indices $j$ and $i$ refer to the involved compounds (BuOH, BTBE and IB). When the adsorption or desorption of a given compound is the reaction ratedetermining step, its activity in Equation 7 or 8 , if included, should be replaced by: $a_{\mathrm{IB}} \rightarrow a_{\mathrm{BTBE}} / a_{\mathrm{BuOH}}, a_{\mathrm{BuOH}} \rightarrow a_{\mathrm{BTBE}} / a_{\mathrm{BB}}, a_{\mathrm{BTBE}} \rightarrow a_{\mathrm{IB}} a_{\mathrm{BuOH}}$

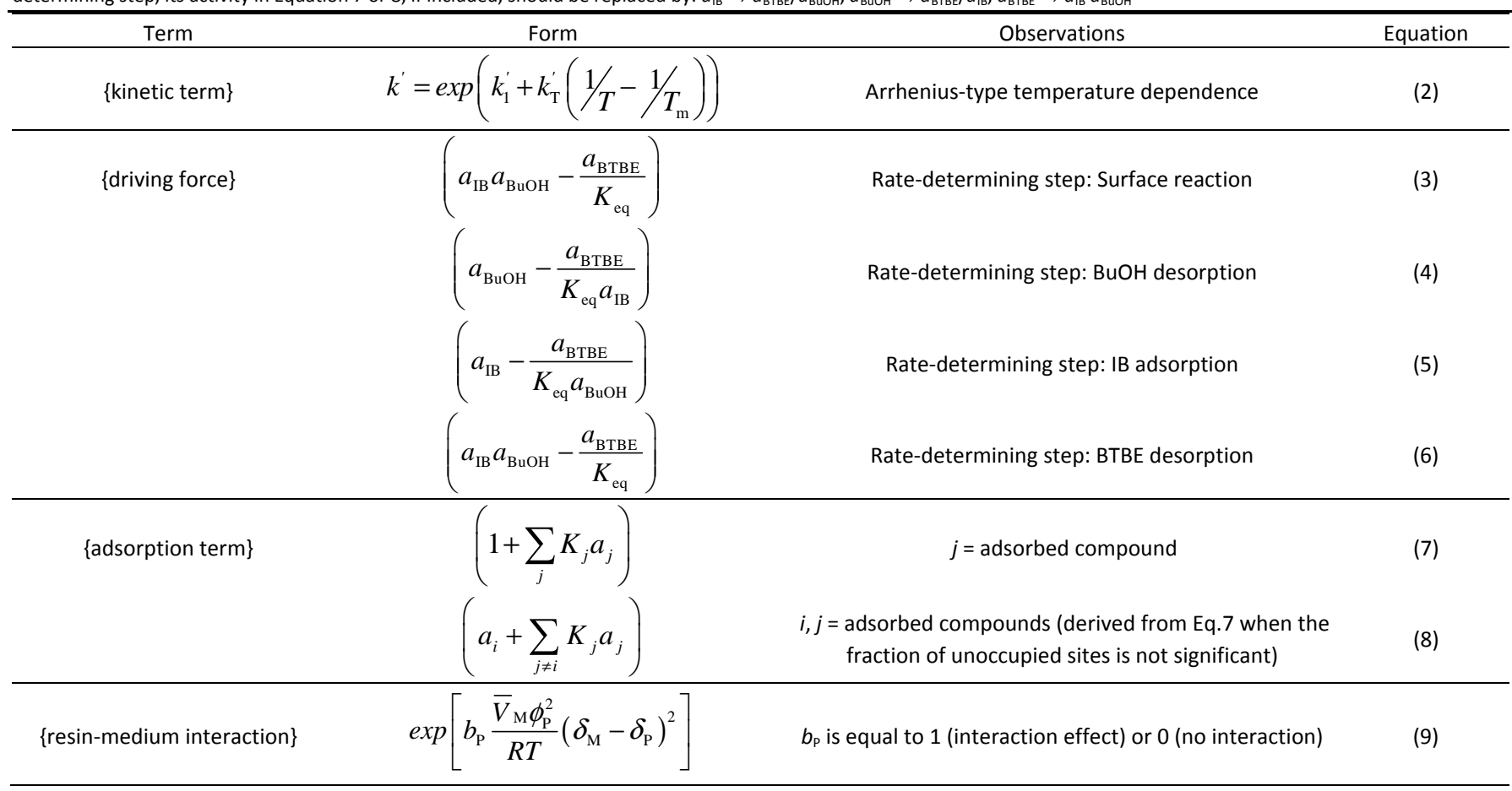


Arrhenius law), adsorption equilibrium constants and the chemical equilibrium constant (both assumed to follow the van't Hoff equation). The particular product depends on the considered mechanism. Parameters $k_{1}^{\prime}$ and $k_{T}^{\prime}$ are the parameters to be fitted. The mean experimental temperature, $T_{\mathrm{m}}$, is included to reduce the correlation between both parameters.

Regarding the driving force term, four different alternatives have been considered, given the considered rate-determining step for the global reaction process, i.e., surface reaction (Equation 3), 1-butanol adsorption (Equation 4), isobutene adsorption (Equation 5), and BTBE desorption (Equation 6). The parameter $K_{\text {eq }}$ in Equations 3 to 6 stands for the chemical equilibrium constant for the synthesis of BTBE, whose value had been determined experimentally in a previous work: ${ }^{7}$

$$
\begin{aligned}
\ln K_{\text {eq }}= & 870.35-\frac{105348}{R T}-\frac{1425.42}{R} \ln T+ \\
& \frac{11.0849}{2 R} T-\frac{28.316 \times 10^{-3}}{6 R} T^{2}+\frac{2.1305 \times 10^{-5}}{12 R} T^{3}
\end{aligned}
$$

In the adsorption term, two different alternatives have been considered: whether the fraction of unoccupied active sites in the catalyst surface affects the reaction rate (Equation 7 ) or the number of vacant active sites is non-significant (Equation 8 , derived from Equation 7 by removing the unity summand, a required mathematical issue to avoid overparameterisation in the model fit). Parameters $K_{j}$ in Equation 7 correspond to the actual adsorption equilibrium constant of each species $j\left(K_{j}=\right.$ $K_{\mathrm{a}, j}$, except when the rate-determining step is a compound adsorption-desorption; then, for that compound, $K_{\mathrm{lB}}=K_{\mathrm{a}, \mathrm{IB}} / K_{\text {eq }}$ or $K_{\mathrm{BuOH}}=K_{\mathrm{a}, \mathrm{BuOH}} / K_{\text {eq }}$, or $\left.K_{\mathrm{BTBE}}=K_{\mathrm{a}, \mathrm{BTBE}} \cdot K_{\text {eq }}\right)$. In Equation 8 , parameters $K_{j}$ are quotients of adsorption equilibrium constants $\left(K_{j}=K_{\mathrm{a}, j} / K_{\mathrm{a}, i}\right.$, except when the rate-determining step is a compound adsorption-desorption; then, for that compound, $K_{\mathrm{lB}}=K_{\mathrm{a}, \mathrm{IB}} /\left(K_{\mathrm{a}, i} \cdot K_{\mathrm{eq}}\right)$, or $K_{\mathrm{BuOH}}=K_{\mathrm{a}, \mathrm{BuOH}} /\left(K_{\mathrm{a}, i} \cdot K_{\text {eq }}\right)$, or $\left.K_{\mathrm{BTBE}}=K_{\mathrm{a}, \mathrm{BTBE}} \cdot K_{\mathrm{eq}} / K_{\mathrm{a}, i}\right)$. For the sake of clarity, Table 2 lists all alternative forms of the adsorption term.

Since $K_{j}$ parameters are adsorption equilibrium constants or a quotient thereof, their temperature dependence has been expressed according to the van't Hoff equation, as follows:

$K_{j}=\exp \left[K_{1, j}+K_{\mathrm{T}, j}\left(1 / T-1 / T_{\mathrm{m}}\right)\right]$

In case that the temperature dependence of $K_{j}$ is not significant, the parameter $K_{\mathrm{T}, j}$ should be taken as equal to zero and, thus, $K_{1, j}$ is the only parameter to be fitted as the $K_{j}$ estimate.

Table 2 Alternative forms of the adsorption term in a LHHW or ER kinetic model for the BTBE synthesis

\begin{tabular}{cccc}
\hline no. & Adsorption term & no. & Adsorption term \\
\hline 1 & 1 & 8 & $1+K_{\mathrm{BuOH}} a_{\mathrm{BuOH}}$ \\
2 & $a_{\mathrm{BuOH}}$ & 9 & $1+K_{\mathrm{BTBE}} a_{\mathrm{BTBE}}$ \\
3 & $a_{\mathrm{IB}}$ & 10 & $1+K_{\mathrm{IB}} a_{\mathrm{IB}}$ \\
4 & $a_{\mathrm{BuOH}}+K_{\mathrm{BTBE}} a_{\mathrm{BTBE}}$ & 11 & $1+K_{\mathrm{BuOH}} a_{\mathrm{BuOH}}+K_{\mathrm{BTBE}} a_{\mathrm{BTBE}}$ \\
5 & $a_{\mathrm{BuOH}}+K_{\mathrm{IB}} a_{\mathrm{IB}}$ & 12 & $1+K_{\mathrm{BuOH}} a_{\mathrm{BuOH}}+K_{\mathrm{IB}} a_{\mathrm{IB}}$ \\
6 & $a_{\mathrm{BTBE}}+K_{\mathrm{IB}} a_{\mathrm{IB}}$ & 13 & $1+K_{\mathrm{BTBE}} a_{\mathrm{BTBE}}+K_{\mathrm{IB}} a_{\mathrm{IB}}$ \\
7 & $a_{\mathrm{BuOH}}+K_{\mathrm{BTBE}} a_{\mathrm{BTBE}}+K_{\mathrm{IB}} a_{\mathrm{IB}}$ & 14 & $1+K_{\mathrm{BuOH}} a_{\mathrm{BuOH}}+K_{\mathrm{BTBE}} a_{\mathrm{BTBE}}+K_{\mathrm{IB}} a_{\mathrm{IB}}$ \\
\hline
\end{tabular}

The exponent $n$ in the adsorption term is related to the number of active sites, or clusters of active sites, involved in the reaction mechanism. Values of $n$ of 1,2 , and 3 are the most likely, according to previous kinetic studies on similar reaction systems. ${ }^{11-13,18,20-22}$

The resin-medium interaction term (Equation 9) accounts for the effect of the reaction medium on the catalyst activity promoted by the difference between the solubility parameters of the reaction medium, $\delta_{\mathrm{M}}$, and the catalyst, $\delta_{\mathrm{P}}$, as observed in analogous reaction systems where the Hildebrand solubility parameter was used. ${ }^{15,23}$ The binary parameter $b_{\mathrm{p}}$ is equal to 0 if the effect of the interaction between the reaction medium and the catalyst is not significant, or equal to 1 if the interaction effect is significant; then, $\delta_{\mathrm{P}}$ could be constant $\left(k_{\mathrm{TP}}=0\right)$ or linearly temperature dependent $\left(k_{\mathrm{TP}} \neq 0\right):^{15,23}$

$\delta_{\mathrm{P}}=k_{\mathrm{P} 1}+k_{\mathrm{PT}}\left(T-T_{\mathrm{m}}\right)$

where both $k_{\mathrm{P} 1}$ and $k_{\mathrm{PT}}$ are the fitting parameters, and the mean temperature $T_{\mathrm{m}}$ is included to reduce the correlation between both parameters. The remaining variables in Equation 9 are the molar volume of the liquid mixture $\bar{V}_{\mathrm{M}}$, estimated from the species concentration and the temperature, ${ }^{24-26}$ the catalyst porosity in the swollen-state $\phi_{p}$, the Hildebrand solubility parameter of the liquid mixture $\delta_{\mathrm{M}}$, estimated as described in the literature, ${ }^{26}$ and the gas constant $R$.

As a result of all possible combinations of the proposed forms of the general kinetic expression terms, a total of 1404 different kinetic equations are obtained, to be fitted individually to the experimental data with the aim of obtaining the best kinetic model.

\section{Experimental section}

\section{Materials}

Reactants. 1-butanol (BuOH, $>99.8 \%$ GC, Sigma-Aldrich), and isobutene (IB) as pure isobutene ( $>99.9 \% \mathrm{GC}$; Air Liquide) or in a synthetic C4 mixture (25\%wt isobutene, $40 \%$ wt isobutane, $35 \% w t$ trans-2-butene; Abelló-Linde). Safety \& hazards: all compounds are flammable, and experiments have been carried out under pressure.

Chemical standards used for chromatographic analysis. 2methyl-2-propanol (TBA, >99.7\% GC, Panreac), 2,4,4-trimethyl1-pentene (TMP1, >98\% GC, Sigma-Aldrich), 2,4,4-trimethyl-2pentene (TMP2, >98\% GC, Sigma-Aldrich). 1-tert-Butoxybutane (butyl tert-butyl ether, BTBE, >98\% GC) was synthesized and purified in our laboratory.

Catalyst. Amberlyst ${ }^{\mathrm{TM}} 35$ (A35, The Dow Chemical Company, now DuPont), a sulfonic macroreticular styrene-divinylbenzene resin. Its physical properties can be found elsewhere. ${ }^{17}$

\section{Apparatus, procedure, and analysis}

Experimental runs were carried out in two different reactor setups. Most of the experiments were run in a batch stirred tank reactor, the rest in a continuously operated fixed-bed catalytic reactor. The purpose of the experiments in the fixedbed reactor was to validate the results in the batch reactor. The initial reaction mixture in batch experiments and the 
reactor feed in continuous experiments did not contain BTBE. The batch reactor experiments were carried out at 2.0 $\mathrm{MPa}$ and at constant temperature, in the range of 318 to $356 \mathrm{~K}$. Initial alcohol to isobutene molar ratio, $R_{\mathrm{BuOH} / \mathrm{BB} \text {, varied }}^{\circ}$ between 1.0 and 2.0, and pure isobutene was used as reactant. The catalytic fixed-bed reactor experiments were carried out at $1.5 \mathrm{MPa}$ and at constant temperature, between 303 and $333 \mathrm{~K}$. The isobutene source was either pure isobutene or the synthetic $\mathrm{C}_{4}$ mixture, with an equimolar alcohol-isobutene mixture at the reactor inlet $\left(R_{\mathrm{BuOH} / \mathrm{BB}}^{\circ}=1.0\right)$. $\mathrm{GC}$ analyses of samples of the reaction medium in both setups allowed quantifying the reactants and products concentrations. Each GC was equipped with a capillary column (HP-PONA 19091S-001, J\&W Scientific, Santa Clara, US; 100\% dimethylpolysiloxane, $50 \mathrm{~m} \times 0.20 \mathrm{~mm} \times 0.50 \mu \mathrm{m}$ ), helium was used as the carrier gas, and the oven temperature was set at $333 \mathrm{~K}$.

A detailed description of the experimental setup and procedure can be found elsewhere. ${ }^{17}$ Further details on the calculation of experimental reaction rates are shown in Section A of the Electronic Supplementary Information, and a list of all experimental conditions and calculated reaction rates in Section B.

\section{Results and discussion}

\section{Experimental results}

In order to focus the present study on the BTBE synthesis kinetics, the experimental conditions were chosen to minimize the side reactions extension, based on previous works on similar systems. ${ }^{24,25}$ Since the amount of byproducts at the end of the experiments was always below $5 \% \mathrm{wt}$, they have been further dismissed from the kinetic analysis. Other side reactions, such as 1-butanol dehydration or further isobutene oligomerisation, were not detected. Obtaining a kinetic model from experimental data requires that the calculated reaction rates are intrinsic kinetics, that is, free from mass and heat transfer effects. The consequence of such effects is to increase the resistance of the global reaction process, so the measured reaction rate would be lower than the reaction rate in the absence of transfer effects. In heterogeneous catalysis, external transfer effects can take place between the fluid bulk phase and the catalyst surface, so they can be avoided by sufficiently reducing the thickness of the film surrounding the catalyst: by increasing the stirring speed in a batch reactor, or by increasing the fluid flow rate in a fixed-bed reactor. In the present reaction system, external mass transfer effects are avoided with stirring speeds of $750 \mathrm{rpm}$ in the batch reactor, and flow rates of $0.031 \mathrm{~g} / \mathrm{s}$ in the fixed-bed setup, as determined in previous works. ${ }^{16,26,27}$

Internal mass transfer effects occur inside the catalyst. They become more noticeable at larger catalyst bead size and at higher reaction temperature. Assuming that there are not external transfer effects, internal mass transfer effects can be easily checked by plotting the logarithm of measured reaction rates obtained at the same composition vs the temperature inverse (Figure 2). If a straight line is obtained, internal mass transfer effects are negligible. For this purpose, a set of experiments was carried out at different temperatures in both reactor systems, i.e., batch and fixed-bed, with the same reactants composition $\left(R_{\mathrm{BuOH} / \mathrm{BB}}^{\circ}=1.0\right)$ in the absence of product, and with $0.25-0.40 \mathrm{~mm}$ catalyst bead size (solid symbols in Figure 2). Experimental points deviate at temperature higher than $333 \mathrm{~K}\left(1 / \mathrm{T}<3.0 \cdot 10^{-3} \mathrm{~K}^{-1}\right.$ in Figure 2$)$, what indicates a significant effect of mass transfer resistances above $333 \mathrm{~K}$. Additional experiments at the same composition and at about $343 \mathrm{~K}$ and $353 \mathrm{~K}$ were carried out using smaller catalyst particles ( $<0.08 \mathrm{~mm}$, open symbols in Figure 2$)$, which resulted well aligned with those obtained at lower temperatures, thus free of mass transfer resistance. In the further kinetic analysis, reaction rates affected by transfer effects were dismissed. From the slope of the solid straight line in Figure 2, the apparent activation energy, $E_{\mathrm{ap}}$, for the BTBE formation reaction has been estimated as $(67 \pm 2) \mathrm{kJ} \mathrm{mol}^{-1}$. This value is in the same range of those quoted in the literature for similar reaction systems using $A 35 .^{10,17,18,28-30}$

\section{Fitting kinetic models to experimental rate data}

The first step in the search of the kinetic model was to fit each of the 1404 built equations to all experimental reaction rates free from mass transfer limitations at once by optimisation of the parameter values. The Levenberg-Marquardt algorithm was used to minimize the residual sum of squares (RSS), defined as:

$R S S=\sum_{i}^{N}\left(r_{\text {exp }}-r_{\text {calc }}\right)_{i}^{2}$

where $r_{\exp }$ is the experimental reaction rate, $r_{\text {calc }}$ is the calculated value, and $N$ is the number of experimental data $(N=136)$. Weighted residual sum of squares, with different

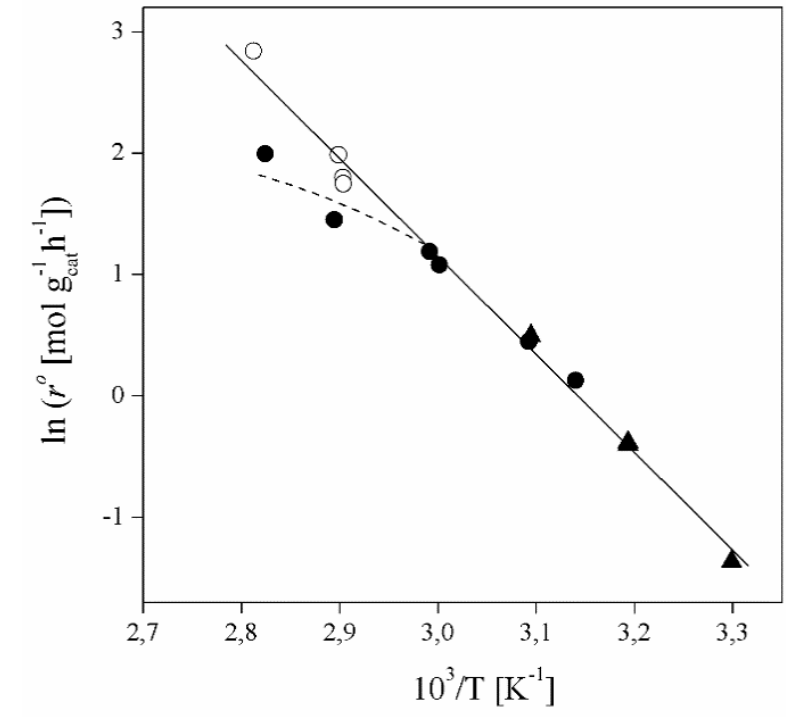

Fig. 2 Arrhenius plot of $B$ TBE formation rates with $\mathrm{R}_{\mathrm{BuOH} / \mathrm{BB}}^{\circ}=1.0$ and pure isobutene. Solid symbols: catalyst bead size $0.25-0.40 \mathrm{~mm}$, batch reactor $(\bullet)$ and fixed-bed reactor $(\boldsymbol{\Delta})$. Open symbols: catalyst bead size $<0.08 \mathrm{~mm}$, batch reactor $(0)$ 
weighting factors, were also tested, resulting in similar model ranking, distribution of residuals and parameter values. Therefore, further analysis refers to RSS.

The discrimination of kinetic models has been carried out by applying the following mathematical and physicochemical criteria to conservatively discard inadequate models:

1. Fitted kinetic equations presenting large RSS values do not provide a satisfactory description of all experimental kinetic data. Figure 3 shows the obtained RSS, where kinetic equations are ordered from lowest to highest RSS values. Consequently, equations presenting RSS values larger than 100 have been rejected.

2. In a suitable kinetic expression, all fitted parameter values should be statistically significant. Their standard error can be estimated from the covariance matrix of the parameters at the optimum. In this regard, models with at least one parameter with an associated standard uncertainty as large as the fitted value have been discarded.

3. Only kinetic equations producing positive values for the apparent activation energy, $E_{\mathrm{ap}}$, can be accepted, because of the experimental evidence that the reaction rate increases with temperature (Figure 2), so models with positive fitted values for $k_{T}^{\prime}$ (Equation 2) are directly discarded. In addition, positive $E_{\text {ap }}$ values clearly inconsistent with previously reported $E_{\mathrm{ap}}$ for similar reaction systems (in the range of 69.3 to $84 \mathrm{~kJ} \mathrm{~mol}^{-1}$ for MTBE, ETBE, PTBE, and TAEE syntheses over A35) $10,17,18,28-$ ${ }^{30}$ can also be rejected. In particular, $E_{\mathrm{ap}}$ values well above $100 \mathrm{~kJ} \mathrm{~mol}^{-1}$ (i.e., $k_{\mathrm{T}}^{\prime}<-13000 \mathrm{~K}^{-1}$ ) or below $45 \mathrm{~kJ} \mathrm{~mol}^{-1}$ (i.e., $k_{T}^{\prime}>-5000 \mathrm{~K}^{-1}$ ) have been rejected.

4. The adsorption process of a given compound on the resin is an exothermic process $\left(\Delta_{\mathrm{a}} H_{j}^{0}<0\right)$. For models with a significant number of unoccupied active sites (Equation 7), $K_{j}$ is the adsorption equilibrium constant of compound $j$, and $K_{\mathrm{T}, j}$ (Equation 11) corresponds to $-\Delta_{\mathrm{a}} H^{\circ} \mathrm{j} / \mathrm{R}$, thus it should be positive. Models whose range of any $K_{\mathrm{T}, j}$ and its

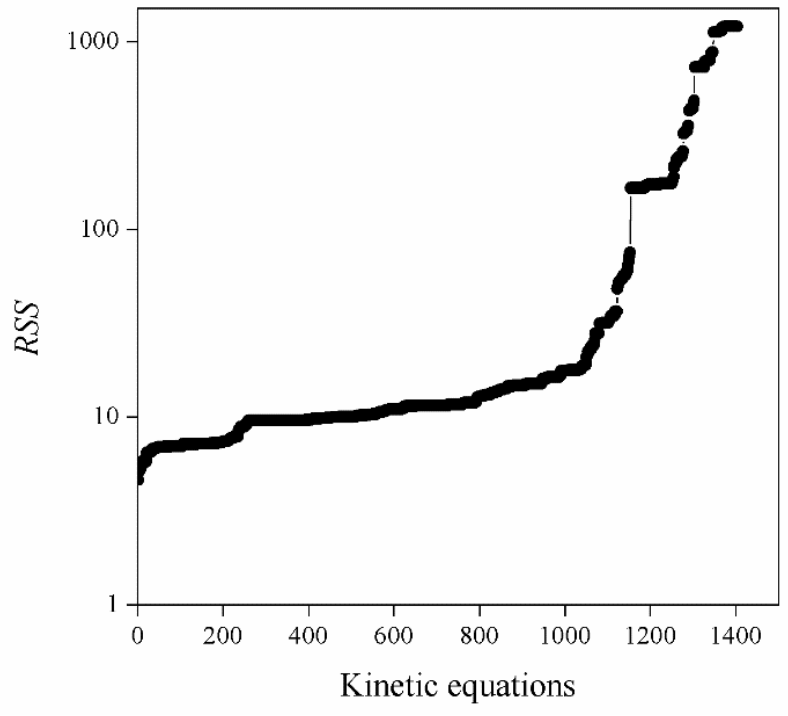

Fig. 3 RSS values of fitted kinetic equations. uncertainty clearly falls in negative values have been discarded. Models where all active sites are considered as occupied (Equation 8) are not affected by this condition, because $K_{j}$ is defined as a ratio of adsorption equilibrium constants and, therefore, $K_{\mathrm{T}, j}=-\left(\Delta_{\mathrm{a}} H^{\circ}-\Delta_{\mathrm{a}} H^{\circ}\right) / \mathrm{R}$, so it can be either positive or negative.

5. From its definition, the resin solubility parameter $\delta_{\mathrm{P}}$ must be positive. In models where $\delta_{\mathrm{p}}$ is temperature dependent, and it is expected to decrease with temperature, ${ }^{19}$ so the range of $k_{\mathrm{PT}}$ and its uncertainty cannot fall completely in positive values. Models that do not meet these conditions have been discarded.

6. The Akaike Information Criterion $(A / C)$, i.e., the relative likelihood of every model, can be used to rank a number of $S$ kinetic equations from more to less plausible upon the basis of robust multimodel inferences. ${ }^{31,32}$ The following expressions apply in the Akaike procedure:

$$
\begin{aligned}
& A I C=m\left[\ln \left(\frac{R S S}{m}\right)\right]+2 k \\
& A I C c=A I C+\frac{2 k(k+1)}{m-k-1} \\
& \Delta_{i}=A I C c_{i}-A I C c_{\min } \\
& w_{i}=\frac{\exp \left(-\frac{1}{2} \Delta_{i}\right)}{\sum_{s=1}^{s} \exp \left(-\frac{1}{2} \Delta_{s}\right)}
\end{aligned}
$$

where $m$ is the number of experimental points, $k$ is the number of parameters in the fitted equation, $A / C C$ is the bias-corrected reduced $A I C$ for situations with low $m / k$ values (i.e., $m / k<40$, like in the present case), $\Delta_{i}$ is an estimator of the level of empirical support for a given model (the lowest $\Delta_{i}$ value corresponds to the most plausible candidate model), $A I C C_{\min }$ is the minimum $A / C C$ among all models, and $w_{i}$ accounts for the relative likelihood of model $i$ out of the $S$ candidate models. ${ }^{31,32}$ Lower $\Delta_{i}$ and higher $w_{i}$ values are an indication of a better model.

The consideration of the mathematical and physicochemical criteria applied in a conservative way allowed to reduce the number of candidate equations from 1404 to only a few. The best ranked models, their optimal parameter values and ranking criteria (RSS, $\Delta_{i}$, and $w_{i}$ ) are listed in Table 3, sorted from best to worst.

A model is considered as substantially supported by empirical evidence when its $\Delta_{i}$ value is lower than $3 .^{31,32}$ The first ranked model (Model 49) stands out as the most plausible one, because of its high probability ( $w_{\text {Model } 49}=93.7 \%$ ) and $\Delta_{\text {Model49 }}=0$ value, far from the second ranked model $\left(w_{\text {Model } 48}=2.1 \%, \Delta_{\text {Model48 }}=8\right)$. In addition, some common features are observed among the first ranked models that support the choice of Model 49: i) none of the equations includes the isobutene contribution in the adsorption term $\left(\Sigma w_{i}=0\right)$, whereas the 1-butanol and BTBE effect appears in the adsorption term for most of the candidate equations $\left(\Sigma w_{i}=0.993\right.$ and $\Sigma w_{i} \approx 1$, respectively); ii) there is a high probability that the first summand in the adsorption term is 
Table 3 Parameter values for the best fitting BTBE kinetic equations. A "- " sign indicates that the related effect is not included in the model.

\begin{tabular}{|c|c|c|c|c|c|c|c|c|c|c|c|c|c|c|c|c|}
\hline \multirow{2}{*}{ Model no. } & \multicolumn{2}{|c|}{$k^{\prime}(\mathrm{mol} / \mathrm{g} \mathrm{h})$} & \multirow{2}{*}{$\begin{array}{l}\text { \{driving } \\
\text { force }^{\text {a }}\end{array}$} & \multicolumn{7}{|c|}{ \{adsorption term\} } & \multicolumn{3}{|c|}{$\delta_{\mathrm{P}}\left(\mathrm{MPa}^{1 / 2}\right)$} & \multirow{2}{*}{ RSS } & \multirow{2}{*}{$\Delta_{i}$} & \multirow{2}{*}{$w_{i}$} \\
\hline & $k_{1}^{\prime}$ & $k_{T}^{\prime}$ & & $1^{\text {st }} \mathrm{Ads}^{\mathrm{b}}$ & $K_{1, \mathrm{BuOH}}$ & $K_{\mathrm{T}, \mathrm{BuOH}}$ & $K_{1, \mathrm{IB}}$ & $K_{\mathrm{T}, \mathrm{IB}}$ & $K_{1, \mathrm{BTBE}}$ & $K_{\mathrm{T}, \mathrm{BTBE}}$ & $n$ & $k_{\mathrm{P} 1}$ & $k_{\mathrm{PT}}$ & & & \\
\hline 49 & 0.320 & -9171 & (a) & $a_{\mathrm{BuOH}}$ & - & - & - & - & -1.119 & -4039 & 1 & 24.51 & - & 6.497 & 0 & 0.937 \\
\hline 290 & 1.917 & -8986 & (a) & 1 & -0.334 & - & - & - & -0.810 & - & 3 & 24.61 & - & 6.948 & 9 & 0.010 \\
\hline 173 & 2.072 & -8994 & (a) & 1 & 0.332 & - & - & - & -0.144 & - & 2 & 24.53 & - & 6.954 & 9 & 0.009 \\
\hline 56 & 3.408 & -9019 & (a) & 1 & 2.951 & - & - & - & 2.491 & - & 1 & 24.32 & - & 6.977 & 10 & 0.007 \\
\hline 978 & 0.412 & -9033 & (c) & 1 & - & - & - & - & -1.178 & - & 3 & 24.06 & - & 7.155 & 11 & 0.004 \\
\hline 291 & 1.975 & -8707 & (a) & 1 & -0.272 & 308 & - & - & -0.804 & - & 3 & 24.60 & - & 6.933 & 11 & 0.004 \\
\hline 861 & 0.428 & -9042 & (c) & 1 & - & - & - & - & -0.761 & - & 2 & 23.94 & - & 7.185 & 11 & 0.003 \\
\hline 166 & 0.088 & -9318 & (a) & $a_{\mathrm{BuOH}}$ & - & - & - & - & -1.655 & -3039 & 2 & 22.56 & - & 7.327 & 16 & $<0.001$ \\
\hline 751 & 0.121 & -9346 & (c) & $a_{\mathrm{BuOH}}$ & - & - & - & - & -0.981 & -3650 & 1 & 22.24 & - & 7.429 & 18 & $<0.001$ \\
\hline 759 & 17.46 & -6477 & (c) & 1 & 17.34 & 2813 & - & - & 16.51 & - & 1 & 22.36 & - & 7.454 & 21 & $<0.001$ \\
\hline 165 & 0.144 & -9196 & (a) & $a_{\mathrm{BuOH}}$ & - & - & - & - & -1.207 & - & 2 & 22.26 & - & 7.749 & 22 & $<0.001$ \\
\hline 2 & 1.170 & -9770 & (a) & 1 & - & - & - & - & - & - & 1 & 20.06 & - & 9.566 & 48 & $<10^{-10}$ \\
\hline
\end{tabular}

${ }^{a}$ Form of the driving force: (a) surface reaction (Equation 3) and (c) isobutene adsorption (Equation 5). ${ }^{b}$ First summand of the adsorption term

$a_{\text {BuOH }}\left(\Sigma w_{i}=0.959\right)$ in front of being $1\left(\Sigma w_{i}=0.041\right)$; iii) the exponent in the adsorption term is likely to be $n=1$ $\left(\Sigma w_{i}=0.966\right) ;$ iv) the resin-medium interaction term is suitable $\left(\Sigma w_{i} \approx 1\right)$; and $\left.v\right)$ the most likely rate-determining step is the surface reaction ( $\Sigma w_{i}=0.993$; driving force type (a) in Table 3 ). Figure 4 shows the adequacy of the prediction of experimental reaction rates by Model 49, the low sensitivity of the model output due to the uncertainty of the fitted parameters and the randomly distributed residuals for each experimental point. Model 49 predicts simultaneously well the experimental data obtained in both batch and fixed-bed reactor systems.

\section{Selected kinetic model}

The proposed kinetic equation Model 49, its parameter estimates, and their standard error are:

$$
r=k^{\prime} \frac{\left(a_{\mathrm{IB}} a_{\mathrm{BuOH}}-\frac{a_{\mathrm{BTBE}}}{K_{\mathrm{Eq}}}\right)}{a_{\mathrm{BuOH}}+K_{\mathrm{BTBE}} a_{\mathrm{BTBE}}} \exp \left(\frac{\bar{V}_{\mathrm{M}} \phi_{\mathrm{P}}^{2}}{R T}\left(\delta_{\mathrm{M}}-\delta_{\mathrm{P}}\right)^{2}\right)
$$

where:

$$
\begin{aligned}
& k^{\prime}\left[\frac{m o l}{g h}\right]=\exp \left[(0.32 \pm 0.02)-(9.17 \pm 0.03) \cdot 10^{3}\left(\frac{1}{T}-\frac{1}{329.4}\right)\right] \\
& K_{\mathrm{BTBE}}=\frac{K_{\mathrm{a}, \mathrm{BTBE}}}{K_{\mathrm{a}, \mathrm{BuOH}}}=\exp \left[-(1.12 \pm 0.06)-(4.0 \pm 0.3) \cdot 10^{3}\left(\frac{1}{T}-\frac{1}{329.4}\right)\right] \\
& \delta_{\mathrm{P}}\left[\mathrm{MPa}^{1 / 2}\right]=(24.51 \pm 0.18)
\end{aligned}
$$

This expression derives from an ER reaction mechanism consisting of the following steps: i) one molecule of 1-butanol adsorbs on a resin active site (or cluster of active sites); ii) it reacts with one isobutene molecule from solution to give one molecule of adsorbed BTBE; and iii) the BTBE molecule
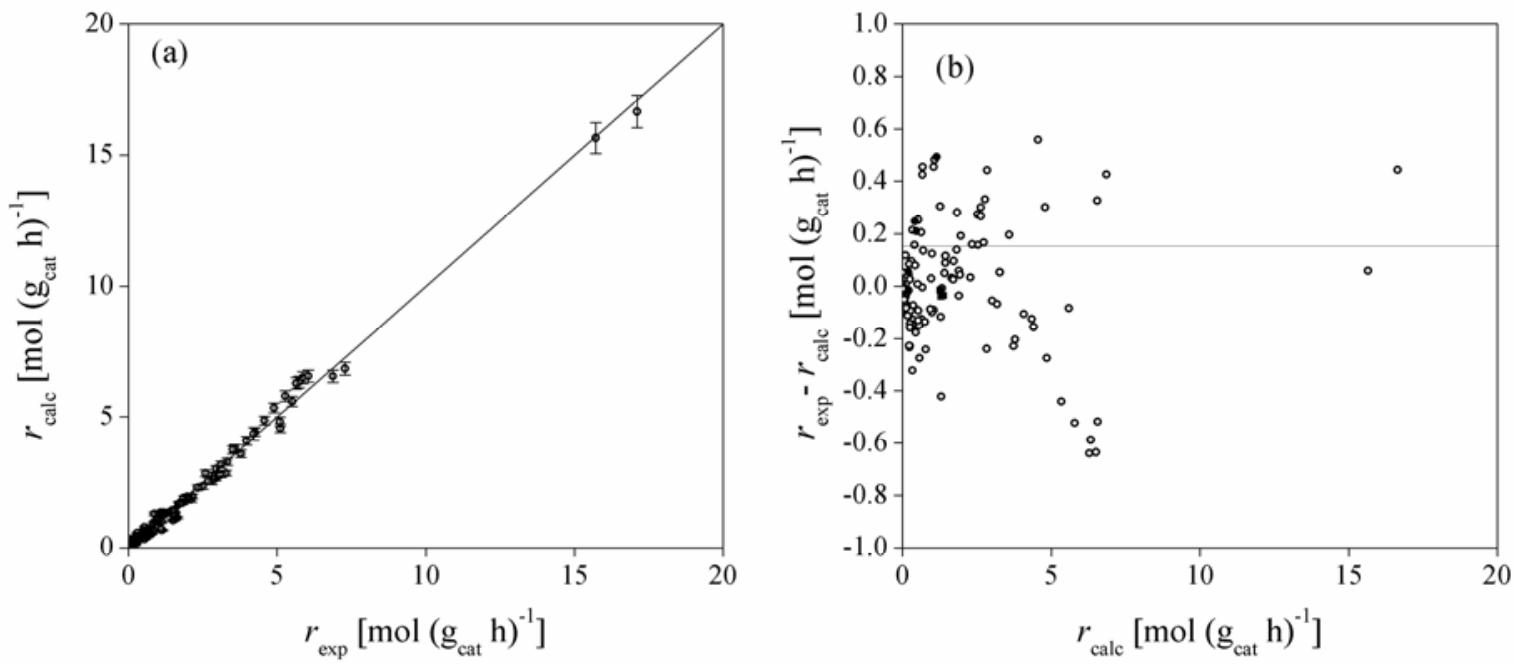

Fig. 4 Comparison between experimental and calculated BTBE reaction rates for Model 49 (a), and residuals distribution (b). Symbols: Experimental rates obtained in the batch reactor $(\circ)$, in the fixed-bed reactor using the $C_{4}$ as isobutene source $(\bullet)$, and in the fixed-bed reactor using pure isobutene $(\square)$. Error bars in (a) correspond to the sensitivity (standard deviation) of the model output due to the parameters uncertainty (see section C in Electronic Supplementary Information). 
desorbs. The surface reaction step is the rate-limiting step of the overall reaction process. This mechanism is in agreement with previous works on analogous reaction systems, e.g., syntheses of $\mathrm{MTBE},{ }^{28} \mathrm{ETBE}^{15}$ or PTBE. ${ }^{10}$

Since the exponent of the adsorption term $n$ is 1 , is equal to the apparent kinetic coefficient $k^{\prime}$ is equal to the intrinsic kinetic constant $k$, as follows:

$$
k^{\prime}=k K_{\mathrm{a}, \mathrm{BuOH}}^{1-n}=k
$$

and, therefore, the true activation energy of the reaction $E_{\mathrm{a}}$ is equal to the apparent one $E_{\mathrm{ap}}$. Since $k_{\mathrm{T}}^{\prime}=-(9.17 \pm 0.03) \cdot 10^{3} \mathrm{~K}$, then $E_{\mathrm{a}}=(76.3 \pm 0.3) \mathrm{kJ} \mathrm{mol}^{-1}$.

The parameter $K_{\mathrm{BTBE}}$ in the adsorption term of Equation 18 corresponds to the ratio between the adsorption equilibrium constants of BTBE and 1-butanol, i.e., $K_{\mathrm{a}, \mathrm{BTBE}} / K_{\mathrm{a}, \mathrm{BuOH}}$. It is related to adsorption thermodynamic properties as follows:

$$
\begin{aligned}
\ln K_{\mathrm{BTBE}} & =\ln \frac{K_{\mathrm{a}, \mathrm{BTBE}}}{K_{\mathrm{a}, \mathrm{BuOH}}}=\frac{-\left(\Delta_{\mathrm{a}} G_{\mathrm{BTBE}}^{\mathrm{o}}-\Delta_{\mathrm{a}} G_{\mathrm{BuOH}}^{\mathrm{o}}\right)}{R T}= \\
& =\frac{-\left(\Delta_{\mathrm{a}} H_{\mathrm{BTBE}}^{\mathrm{o}}-\Delta_{\mathrm{a}} H_{\mathrm{BuOH}}^{\mathrm{o}}\right)}{R T} \frac{1}{T}+\frac{\Delta_{\mathrm{a}} S_{\mathrm{BTBE}}^{\mathrm{o}}-\Delta_{\mathrm{a}} S_{\mathrm{BuOH}}^{\mathrm{o}}}{R}
\end{aligned}
$$

where $K_{\mathrm{a}, \mathrm{BTBE}}$ is the adsorption equilibrium constant of BTBE, and $\Delta_{a} G^{\circ}, \Delta_{a} H^{\circ}{ }_{j}$ and $\Delta_{a} S^{\circ}$ are the adsorption Gibbs free energy, enthalpy and entropy changes of compound $j$, respectively. The adsorption enthalpy and entropy changes of individual compounds cannot be obtained from the parameter estimates of the kinetic model. Instead, parameters provide differences between BTBE and 1-butanol adsorption enthalpy and entropy

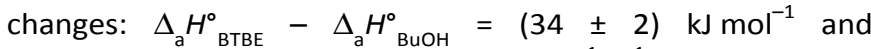
$\Delta_{a} S_{\text {BTBE }}^{\circ}-\Delta_{a} S_{\text {BuOH }}^{\circ}=(93 \pm 7) \mathrm{J} \mathrm{mol}^{-1} \mathrm{~K}^{-1}$. Consequently, 1-butanol adsorption on the resin is more exothermic than BTBE adsorption, and the entropic loss due to adsorption for 1-butanol is larger than for BTBE. A positive difference between the adsorption enthalpies is consistent with values reported in a previous work on adsorption equilibrium of BTBE and 1-butanol on A35, but in the gas phase: $\Delta_{\mathrm{a}} H^{\circ}{ }_{\text {BTBE }}=-21.8 \mathrm{~kJ} \mathrm{~mol}^{-1}$ and $\Delta_{\mathrm{a}} H^{\circ}{ }_{\text {BUOH }}=-41.8 \mathrm{~kJ} \mathrm{~mol}^{-1} .{ }^{33}$

The estimate of the Hildebrand solubility parameter of $A 35, \delta_{\mathrm{P}}$ (Equation 12), can be considered as constant in the assayed temperature range and equal to $(24.51 \pm 0.18) \mathrm{MPa}^{1 / 2}$. This value is slightly larger than the published $\delta_{\mathrm{p}}$ values for this catalyst in similar reaction systems, also being constant: $(20.5$ $\pm 0.3) \mathrm{MPa}^{1 / 2}$ in the PTBE synthesis, ${ }^{10}(20.9 \pm 2.0) \mathrm{MPa}^{1 / 2}$ in the ETBE synthesis, ${ }^{29}$ and (21.16 \pm 0.12$) \mathrm{MPa}^{1 / 2}$ in the simultaneous synthesis of ETBE and TAEE. ${ }^{17}$ However, it fully agrees with the Hildebrand solubility parameter estimated by means of the Hoftyzer and van Krevelen group contribution method, ${ }^{34}$ resulting in $\delta_{\mathrm{P}}=25.45 \mathrm{MPa}^{1 / 2}$ at $298 \mathrm{~K}$. The Hansen solubility parameter, which is considered as a refinement of the Hildebrand parameter, has also been estimated from group contribution, ${ }^{33}$ resulting in $\delta_{\mathrm{P}}=25.54 \mathrm{MPa}^{1 / 2}$ at $298 \mathrm{~K}$. With the aim of checking whether the choice of the selected model, and thus the reaction mechanism, would be affected in case that $\delta_{\mathrm{p}}$ were roughly estimated, all kinetic equations have been fitted again to experimental rate data, but taking $\delta_{\mathrm{p}}$ as a fixed value equal to $20.85 \mathrm{MPa}^{1 / 2}$ (the average of the estimated values quoted in literature $)^{10,17,29}$ if appearing in the model. The best ranked models obtained with this constraint are listed in Table S2 (section D in Electronic Supplementary information). Globally, the residual sum of squares (RSS) is now slightly higher due to the reduction of the degrees of freedom of the fit, but the group of best ranked models is coincident with the non-restricted case, and Model 49 is again the most plausible one $\left(w_{\text {Model } 49}=44.7 \%\right.$, followed by $w_{\text {Model } 166}$ $=8.9 \%)$. This result reinforces the choice of the selected kinetic equation Model 49 as the best one.

\section{Conclusions}

The kinetics of the liquid-phase etherification reaction of isobutene with 1-butanol to produce butyl tert-butyl ether using Amberlyst ${ }^{\mathrm{TM}} 35$ as the catalyst has been studied at 303$356 \mathrm{~K}$ in the liquid phase. Significant internal mass transfer limitations have been detected at temperatures above $333 \mathrm{~K}$ using $0.25-0.40 \mathrm{~mm}$ catalyst bead size, but this effect was not noticeable for bead size smaller than $0.08 \mathrm{~mm}$. Reaction rates free from mass transfer effects obtained in two reaction setups (a batch reactor and a differential tubular reactor) have been used to fit a large number of candidate kinetic equations, each derived from a different mechanism and ratedetermining step, based on the Langmuir-HinshelwoodHougen-Watson and Eley-Rideal formalisms. The possible effects of the reaction medium on the catalyst activity, and of the temperature on the parameters of the kinetic equations have also been considered. Discrimination of models has been accomplished by applying mathematical and physicochemical criteria in a conservative manner. The Akaike Information Criterion has been used to rank the reliability of the kinetic models. The best model is derived from an Eley-Rideal type mechanism, where one molecule of 1-butanol adsorbed on one active site reacts with one isobutene molecule from the liquid phase to form one BTBE molecule that, finally, desorbs. The rate-determining step is the surface reaction. The adsorption of 1-butanol on the resin is more exothermic than BTBE adsorption. The interaction between the reaction medium and the resin has a significant effect on the catalytic activity.

\section{Conflicts of interest}

There are no conflicts to declare.

\section{Acknowledgements}

The authors thank The Dow Chemical Company, now DuPont, for providing the ion exchange resin used as the catalyst in this work.

\section{Notes and references}

1 J. Zheng, Y. Tashiro, Q. Wang and K. Sonomoto, J. Biosci. Bioeng., 2015, 119, 1.

2 B. Ndaba, I. Chiyanzu and S. Marx, Biotechnol. Reports, 2015, 8,1 . 
3 A. Démolis, M. Eternot, N. Essayem and F. Rataboul, New J. Chem., 2016, 40, 3747.

4 G.W. Huber, S. Iborra and A. Corma, Chem. Rev., 2006, 106, 4044.

5 K.G. Sharonov, Y.B. Mishentseva, A.M. Rozhnov, E.A. Miroshnichenko and L.I. Korchatova, J. Chem. Thermodyn., 1991, 23, 141.

6 K.G. Sharonov, Y.B. Mishentseva, A.M. Rozhnov, E.A. Miroshnichenko and L.I. Korchatova, J. Chem. Thermodyn. 1991, 23, 636.

7 J.H. Badia, C. Fité, R. Bringué, E. Ramírez and F. Cunill, J. Chem. Eng. Data, 2016, 61, 1054.

8 F. Ancillotti, M. Massi Mauri and E. Pescarollo, J. Catal., 1977, 46, 49

9 F. Ancillotti, M. Massi Mauri, E. Pescarollo, L. Romagnoni, J. Mol. Catal. 4 (1978) 37-48.

10 A. Gicquel, B. Torck, J. Catal. 1983, 83, 9.

11 O. Françoisse, F.C. Thyrion, Chem. Eng. Process., 1991, 30, 141.

12 A. Rehfinger and U. Hoffmann, Chem. Eng. Sci., 1990, 45, 1605.

13 C. Fité, M. Iborra, J. Tejero, J.F. Izquierdo and F. Cunill, Ind. Eng. Chem. Res., 1994, 33, 581.

14 D. Parra, J. Tejero, F. Cunill, M. Iborra and J.F. Izquierdo, Chem. Eng. Sci., 1994, 49, 4563.

15 C. Fité, J. Tejero, M. Iborra, F. Cunill and J.F. Izquierdo, AIChE J., 1998, 44, 2273.

16 K. Sundmacher, R.S. Zhang, U. Hoffmann, Chem. Eng. Technol., 1995, 18, 269.

17 J.H. Badia, C. Fité, R. Bringué, M. Iborra and F. Cunill, Chem. Eng. J., 2019, 356, 219.

18 R. Soto, C. Fité, E. Ramírez, R. Bringué and F. Cunill, Chem. Eng. J., 2017, 307, 122.

19 D. Constantinescu and J. Gmehling, J. Chem. Eng. Data, 2016, 61, 2738

20 A.M. Al-Jarallah, M.A.B. Siddiqui and A.K.K. Lee, Can. J. Chem. Eng., 1988, 66, 802.

21 J.A. Linnekoski, A.O. Krause and L.K. Rihko, Ind. Eng. Chem. Res., 1997, 36, 310.

22 G. Bozga, A. Motelica, R. Dima, V. Plesu, A. Toma and C. Simion, Chem. Eng. Process., 2008, 47, 2247.

23 C. Fité, J. Tejero, M. Iborra, F. Cunill, J.F. Izquierdo and D. Parra, Appl. Catal. A Gen., 1998, 169, 165.

24 R.C. Reid, J.M. Prausnitz and B.E. Poling, The Properties of Gases and Liquids, fourth ed., McGraw-Hill, New York, 1987.

25 B.E. Poling, J.M. Prausnitz, J.P. O'Connell and R.C. Reid, The Properties of Gases and Liquids, fifth ed., McGraw-Hill, New York, 2001.

26 C.L. Yaws, Thermophysical Properties of Chemicals and Hydrocarbons, William Andrew Inc., Norwich, NY, 2008.

27 M. Vila, F. Cunill, J.-F. Izquierdo, J. González and A. Hernández, Appl. Catal. A Gen., 1994, 117, L99.

28 J.H. Badia, C. Fité, R. Bringué, E. Ramírez and F. Cunill, Appl. Catal. A Gen., 2013, 468, 384.

29 J.H. Badia, C. Fité, R. Bringué, E. Ramírez and M. Iborra, J. Ind. Eng. Chem., 2016, 42, 36.

30 M. Umar, A.R. Saleemi and S. Qaiser, Catal. Commun., 2008, 9, 721.

31 R. González, Performance of Amberlyst ${ }^{T M} 35$ in the Synthesis of ETBE from Ethanol and $C_{4}$ Cuts (Ph.D. Thesis), University of Barcelona, 2011.

32 Ó. Santín, Estudio del control de las etapas físicas en las sintesis de MTBE y ETBE (M.Sc. Thesis), University of Barcelona, 2005.

33 M.R.E. Symonds and A. Moussalli, Behav. Ecol. Sociobiol., 2011, 65, 13.
34 K.P. Burnham and D.R. Anderson, Model Selection and Multimodel Inference: A Practical Information-Theoretic Approach, second ed., Springer-Verlag, New York, 2002.

35 R. Soto, N. Oktar, C. Fité, E. Ramírez, R. Bringué and J. Tejero, Chem. Eng. Technol., 2017, 40, 889.

36 D.W. van Krevelen and K. te Nijenhuis, Properties of Polymers: Their Correlation with Chemical Structure; their Numerical Estimation and Prediction from Additive Group Contributions, fourth ed., Elsevier Science, Amsterdam, The Netherlands, 2009. 


\section{Liquid-phase synthesis of butyl tert-butyl ether catalysed by ion- exchange resins: kinetic modelling through in-depth model discrimination}

Jordi Hug Badia, ${ }^{a}$ Carles Fité, ${ }^{* a}$ Roger Bringué, ${ }^{a}$ Eliana Ramírez, ${ }^{a}$ Montserrat Iborra ${ }^{a}$

Chemical Engineering and Analytical Chemistry Department, Faculty of Chemistry, University of Barcelona, Martí i Franquès 1-11, 08028 Barcelona, Spain

*Corresponding author. Tel.: +34 93402 1304; Fax: +34 93402 1291; E-mail address: fite@ub.edu

\section{Electronic Supplementary Information (ESI)}

\section{A. Calculation of experimental reaction rates}

In the present work, two different reactor setups have been used: a fixed-bed reactor and a batch reactor. In the fixed-bed reactor, one experimental run provides only one measured reaction rate, calculated from the composition change between the reactor inlet and at outlet at the steady state. Since the reactor operated under differential regime, i.e., at low conversion, the reaction rate can be assumed to be constant along the reactor. The experimental isobutene consumption rate was calculated as follows:

$-r_{\mathrm{IB}}=\frac{F_{\mathrm{IB}}^{\mathrm{o}}\left(X_{\mathrm{IB}, \text { outlet }}-X_{\mathrm{IB}, \text { inlet }}\right)}{W_{\text {cat }}}$

where $F_{\mathrm{IB}}^{0}$ is the isobutene molar flow rate at null conversion, $X_{\mathrm{IB}, \text { outlet }}$ is the isobutene conversion at the reactor outlet, and $X_{\mathrm{IB} \text {,inlet }}$ is the inlet isobutene conversion, and $W_{\text {cat }}$ is the mass of dry catalyst in the reactor bed. In particular, the reactor inlet stream was only composed of reactants and, therefore, $X_{\mathrm{IB}, \text { inlet }}=0$.

In the batch reactor system, the evolution of the reaction medium composition was obtained from samples taken at different times. For each experimental run, the progress of the isobutene molar content was used to fit an empirical function that allowed calculating its slope at the sampled times, which is related with the experimental formation rate of compound $j, r_{j}$, as follows:

$r_{j}=\frac{1}{W_{\text {cat }}}\left(\frac{d n_{j}}{d t}\right)$

where $W_{\text {cat }}$ is the mass of dry catalyst, $n_{j}$ is the number of moles of compound $j$ and $t$ is the reaction time.

As an illustrative example, Figure S1 shows the result of two different experimental runs carried out in the batch reactor system and how the experimental reaction rate was calculated at instants where samples were taken. 


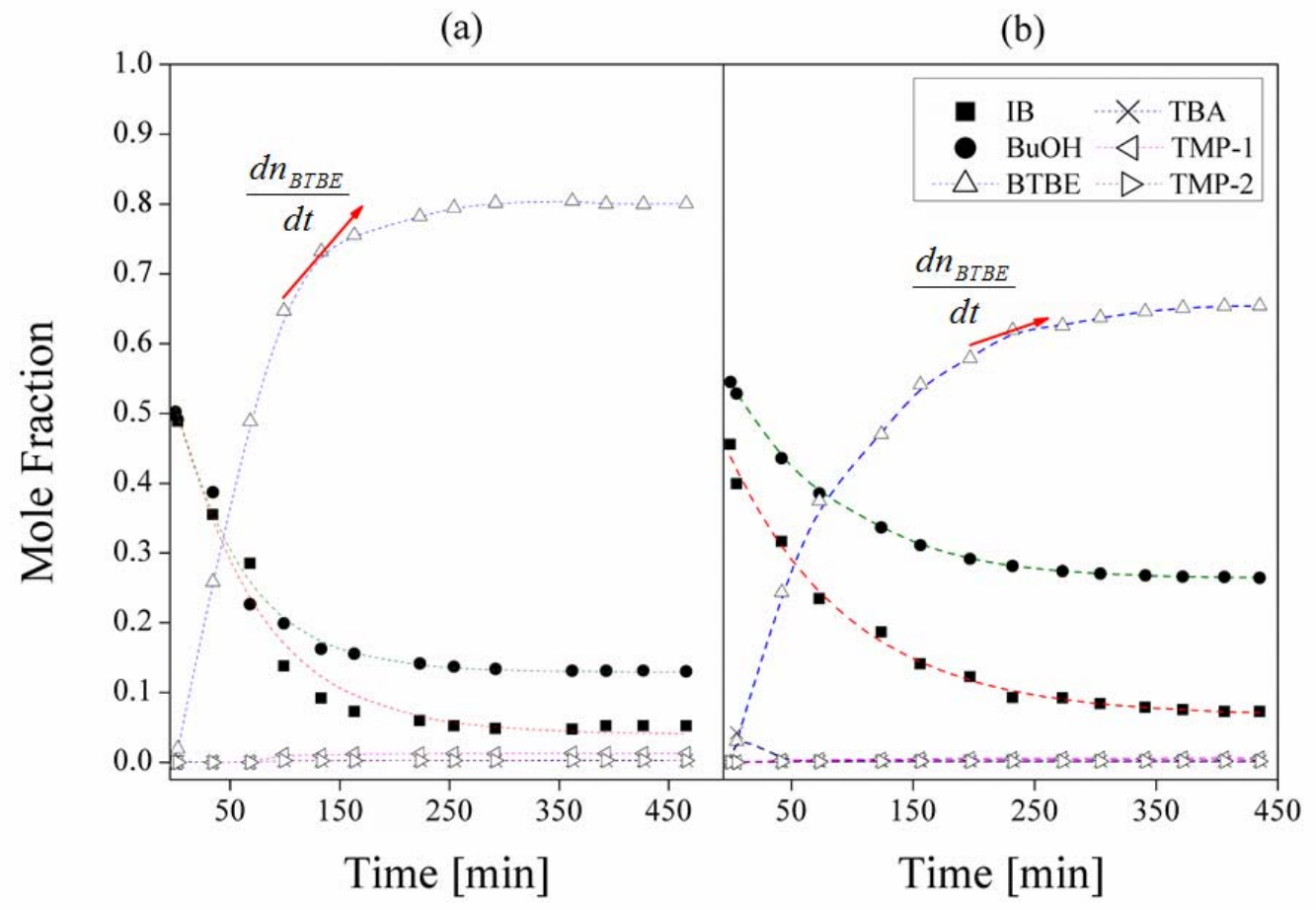

Fig. S1 Experimental mole evolution obtained in the batch reactor system under two different conditions: (a) $\mathrm{T}=323.4 \mathrm{~K}$, $R_{\text {Buон//B }}^{\circ}=1.0 ;$ (b) $\mathrm{T}=343.5 \mathrm{~K}, R_{\text {Buон//B }}^{\circ}=1.2$. Dashed lines correspond to fitted empirical equation. Red arrows represent the derivative with respect to time, used to calculate experimental reaction rates at those instants. 


\section{B. Experimental conditions and calculated reaction rates}

Table S1. Experimental conditions and calculated experimental rates considered in the fit of the kinetic equations

\begin{tabular}{|c|c|c|c|c|c|c|c|c|c|c|c|c|c|}
\hline \multirow{2}{*}{$\begin{array}{l}\text { Exper. } \\
\text { setup }\end{array}$} & \multirow{2}{*}{$\begin{array}{c}T \\
(\mathrm{~K})\end{array}$} & \multirow{2}{*}{$\begin{array}{c}t \\
(\min )\end{array}$} & \multirow{2}{*}{$\begin{array}{c}W_{\text {cat }} \\
\text { (g) }\end{array}$} & \multicolumn{9}{|c|}{ Mole fraction } & \multirow{2}{*}{$\begin{array}{c}r_{\mathrm{BTBE}} \\
{[\mathrm{mol} / \mathrm{g} \mathrm{h}]}\end{array}$} \\
\hline & & & & $x_{1 \mathrm{~B}}$ & $\boldsymbol{X}_{\text {BuOH }}$ & $\boldsymbol{x}_{\mathrm{BTBE}}$ & $\boldsymbol{x}_{\mathrm{TBA}}$ & $\boldsymbol{X}_{\text {TMP-1 }}$ & $x_{\text {TMP-2 }}$ & $\boldsymbol{X}_{i \text {-butane }}$ & $\begin{array}{c}\boldsymbol{x}_{\text {trans-2- }} \\
\text { butene }\end{array}$ & $\begin{array}{l}\boldsymbol{x}_{\text {cis-2- }} \\
\text { butene }\end{array}$ & \\
\hline \multirow{7}{*}{$\begin{array}{l}\text { Plug- } \\
\text { flow } \\
\text { reactor }\end{array}$} & 333.15 & - & 0.0810 & 0.203 & 0.135 & 0 & 0 & 0 & 0 & 0.332 & 0.328 & 0.002 & 1.129 \\
\hline & 333.15 & - & 0.0420 & 0.193 & 0.180 & 0 & 0 & 0 & 0 & 0.313 & 0.313 & 0.002 & 1.031 \\
\hline & 333.15 & - & 0.0421 & 0.190 & 0.193 & 0 & 0 & 0 & 0 & 0.311 & 0.305 & 0.002 & 1.212 \\
\hline & 313.15 & - & 0.1685 & 0.488 & 0.512 & 0 & 0 & 0 & 0 & 0 & 0 & 0 & 0.666 \\
\hline & 313.15 & - & 0.2080 & 0.520 & 0.480 & 0 & 0 & 0 & 0 & 0 & 0 & 0 & 0.680 \\
\hline & 303.15 & - & 0.0440 & 0.548 & 0.452 & 0 & 0 & 0 & 0 & 0 & 0 & 0 & 0.255 \\
\hline & 323.15 & - & 0.0217 & 0.490 & 0.510 & 0 & 0 & 0 & 0 & 0 & 0 & 0 & 1.640 \\
\hline \multirow{14}{*}{$\begin{array}{l}\text { Batch } \\
\text { reactor }\end{array}$} & 323.35 & 0 & 0.7200 & 0.498 & 0.502 & 0 & 0 & 0 & 0 & 0 & 0 & 0 & 1.561 \\
\hline & 323.35 & 2 & & 0.488 & 0.491 & 0.019 & 0.001 & 0 & 0 & 0 & 0 & 0 & 1.506 \\
\hline & 323.35 & 34 & & 0.355 & 0.387 & 0.258 & 0.000 & 0 & 0 & 0 & 0 & 0 & 0.844 \\
\hline & 323.35 & 68 & & 0.285 & 0.226 & 0.489 & 0.000 & 0 & 0 & 0 & 0 & 0 & 0.457 \\
\hline & 323.35 & 99 & & 0.138 & 0.199 & 0.647 & 0.002 & 0.011 & 0.002 & 0 & 0 & 0 & 0.261 \\
\hline & 323.35 & 133 & & 0.092 & 0.162 & 0.732 & 0.002 & 0.010 & 0.002 & 0 & 0 & 0 & 0.141 \\
\hline & 323.35 & 163 & & 0.073 & 0.155 & 0.756 & 0.002 & 0.012 & 0.002 & 0 & 0 & 0 & 0.082 \\
\hline & 323.35 & 223 & & 0.059 & 0.142 & 0.782 & 0.002 & 0.012 & 0.002 & 0 & 0 & 0 & 0.028 \\
\hline & 323.35 & 254 & & 0.052 & 0.137 & 0.794 & 0.002 & 0.012 & 0.002 & 0 & 0 & 0 & 0.016 \\
\hline & 323.35 & 292 & & 0.048 & 0.134 & 0.801 & 0.002 & 0.012 & 0.003 & 0 & 0 & 0 & 0.008 \\
\hline & 323.35 & 362 & & 0.047 & 0.131 & 0.804 & 0.002 & 0.013 & 0.003 & 0 & 0 & 0 & 0.002 \\
\hline & 323.35 & 393 & & 0.052 & 0.131 & 0.800 & 0.002 & 0.012 & 0.003 & 0 & 0 & 0 & 0.001 \\
\hline & 323.35 & 427 & & 0.052 & 0.131 & 0.800 & 0.002 & 0.013 & 0.003 & 0 & 0 & 0 & 0.001 \\
\hline & 323.35 & 466 & & 0.052 & 0.130 & 0.800 & 0.002 & 0.012 & 0.003 & 0 & 0 & 0 & 0.000 \\
\hline \multirow{18}{*}{$\begin{array}{l}\text { Batch } \\
\text { reactor }\end{array}$} & 318.45 & 0 & 0.5100 & 0.492 & 0.508 & 0 & 0 & 0 & 0 & 0 & 0 & 0 & 1.261 \\
\hline & 318.45 & 3 & & 0.489 & 0.503 & 0.008 & 0.001 & 0 & 0 & 0 & 0 & 0 & 1.217 \\
\hline & 318.45 & 33 & & 0.403 & 0.436 & 0.150 & 0.002 & 0.009 & 0.002 & 0 & 0 & 0 & 0.853 \\
\hline & 318.45 & 63 & & 0.316 & 0.392 & 0.268 & 0.002 & 0.017 & 0.004 & 0 & 0 & 0 & 0.599 \\
\hline & 318.45 & 93 & & 0.246 & 0.343 & 0.379 & 0.002 & 0.024 & 0.005 & 0 & 0 & 0 & 0.420 \\
\hline & 318.45 & 125 & & 0.208 & 0.311 & 0.445 & 0.002 & 0.028 & 0.006 & 0 & 0 & 0 & 0.288 \\
\hline & 318.45 & 156 & & 0.180 & 0.272 & 0.509 & 0.002 & 0.031 & 0.007 & 0 & 0 & 0 & 0.199 \\
\hline & 318.45 & 187 & & 0.143 & 0.252 & 0.564 & 0.002 & 0.032 & 0.007 & 0 & 0 & 0 & 0.138 \\
\hline & 318.45 & 217 & & 0.120 & 0.229 & 0.608 & 0.002 & 0.033 & 0.007 & 0 & 0 & 0 & 0.097 \\
\hline & 318.45 & 248 & & 0.107 & 0.219 & 0.631 & 0.002 & 0.034 & 0.007 & 0 & 0 & 0 & 0.067 \\
\hline & 318.45 & 287 & & 0.087 & 0.193 & 0.684 & 0.002 & 0.028 & 0.006 & 0 & 0 & 0 & 0.042 \\
\hline & 318.45 & 313 & & 0.068 & 0.196 & 0.692 & 0.002 & 0.035 & 0.008 & 0 & 0 & 0 & 0.031 \\
\hline & 318.45 & 345 & & 0.058 & 0.190 & 0.707 & 0.002 & 0.035 & 0.008 & 0 & 0 & 0 & 0.021 \\
\hline & 318.45 & 450 & & 0.043 & 0.184 & 0.727 & 0.002 & 0.036 & 0.008 & 0 & 0 & 0 & 0.006 \\
\hline & 318.45 & 480 & & 0.041 & 0.179 & 0.734 & 0.002 & 0.036 & 0.008 & 0 & 0 & 0 & 0.004 \\
\hline & 318.45 & 547 & & 0.039 & 0.179 & 0.736 & 0.002 & 0.037 & 0.008 & 0 & 0 & 0 & 0.002 \\
\hline & 318.45 & 580 & & 0.037 & 0.177 & 0.740 & 0.002 & 0.035 & 0.008 & 0 & 0 & 0 & 0.001 \\
\hline & 318.45 & 621 & & 0.035 & 0.177 & 0.742 & 0.002 & 0.036 & 0.008 & 0 & 0 & 0 & 0.001 \\
\hline \multirow{13}{*}{$\begin{array}{l}\text { Batch } \\
\text { reactor }\end{array}$} & 344.95 & 0 & 0.1296 & 0.492 & 0.508 & 0 & 0 & 0 & 0 & 0 & 0 & 0 & 8.116 \\
\hline & 344.95 & 3 & & 0.480 & 0.501 & 0.017 & 0.001 & 0.001 & 0 & 0 & 0 & 0 & 7.653 \\
\hline & 344.95 & 19 & & 0.396 & 0.463 & 0.124 & 0.001 & 0.013 & 0.003 & 0 & 0 & 0 & 5.593 \\
\hline & 344.95 & 35 & & 0.329 & 0.420 & 0.225 & 0.001 & 0.020 & 0.005 & 0 & 0 & 0 & 4.088 \\
\hline & 344.95 & 51 & & 0.261 & 0.381 & 0.325 & 0.001 & 0.025 & 0.007 & 0 & 0 & 0 & 2.988 \\
\hline & 344.95 & 66 & & 0.212 & 0.356 & 0.395 & 0.001 & 0.028 & 0.007 & 0 & 0 & 0 & 2.227 \\
\hline & 344.95 & 82 & & 0.168 & 0.341 & 0.452 & 0.002 & 0.029 & 0.008 & 0 & 0 & 0 & 1.628 \\
\hline & 344.95 & 100 & & 0.149 & 0.313 & 0.498 & 0.001 & 0.031 & 0.008 & 0 & 0 & 0 & 1.144 \\
\hline & 344.95 & 116 & & 0.118 & 0.298 & 0.543 & 0.001 & 0.031 & 0.008 & 0 & 0 & 0 & 0.836 \\
\hline & 344.95 & 138 & & 0.094 & 0.286 & 0.577 & 0.002 & 0.033 & 0.009 & 0 & 0 & 0 & 0.543 \\
\hline & 344.95 & 168 & & 0.084 & 0.281 & 0.591 & 0.001 & 0.033 & 0.009 & 0 & 0 & 0 & 0.302 \\
\hline & 344.95 & 187 & & 0.071 & 0.288 & 0.599 & 0.001 & 0.033 & 0.008 & 0 & 0 & 0 & 0.208 \\
\hline & 344.95 & 220 & & 0.074 & 0.274 & 0.608 & 0.001 & 0.033 & 0.009 & 0 & 0 & 0 & 0.109 \\
\hline
\end{tabular}




\begin{tabular}{|c|c|c|c|c|c|c|c|c|c|c|c|c|c|}
\hline \multirow{4}{*}{$\begin{array}{l}\text { Exper. } \\
\text { setup }\end{array}$} & \multirow{2}{*}{$\begin{array}{c}T \\
(\mathrm{~K})\end{array}$} & \multirow{2}{*}{$\begin{array}{c}t \\
(\min )\end{array}$} & \multirow{4}{*}{$\begin{array}{c}W_{\text {cat }} \\
\text { (g) }\end{array}$} & \multicolumn{9}{|c|}{ Mole fraction } & \multirow{2}{*}{$\begin{array}{c}r_{\text {BTBE }} \\
{[\mathrm{mol} / \mathrm{g} \mathrm{h}]}\end{array}$} \\
\hline & & & & $x_{1 B}$ & $\boldsymbol{X}_{\mathrm{BuOH}}$ & $\boldsymbol{x}_{\mathrm{BTBE}}$ & $\boldsymbol{x}_{\text {TBA }}$ & $\boldsymbol{x}_{\text {TMP-1 }}$ & $\boldsymbol{X}_{\mathrm{TMP}-2}$ & $\boldsymbol{X}_{\boldsymbol{i} \text {-butane }}$ & $\begin{array}{c}\boldsymbol{X}_{\text {trans-2- }} \\
\text { butene }\end{array}$ & $\begin{array}{l}\boldsymbol{X}_{\text {cis-2- }} \\
\text { butene }\end{array}$ & \\
\hline & 344.95 & 237 & & 0.073 & 0.273 & 0.611 & 0.001 & 0.033 & 0.009 & 0 & 0 & 0 & 0.078 \\
\hline & 344.95 & 254 & & 0.065 & 0.268 & 0.622 & 0.001 & 0.035 & 0.009 & 0 & 0 & 0 & 0.056 \\
\hline \multirow{20}{*}{$\begin{array}{l}\text { Batch } \\
\text { reactor }\end{array}$} & 344.35 & 0 & \multirow[t]{20}{*}{0.0353} & 0.478 & 0.522 & 0 & 0 & 0 & 0 & 0 & 0 & 0 & 7.434 \\
\hline & 344.35 & 4 & & 0.476 & 0.521 & 0.001 & 0.002 & 0 & 0 & 0 & 0 & 0 & 7.273 \\
\hline & 344.35 & 20 & & 0.451 & 0.517 & 0.009 & 0.022 & 0 & 0 & 0 & 0 & 0 & 6.664 \\
\hline & 344.35 & 37 & & 0.431 & 0.504 & 0.033 & 0.031 & 0 & 0 & 0 & 0 & 0 & 6.073 \\
\hline & 344.35 & 53 & & 0.409 & 0.488 & 0.064 & 0.038 & 0 & 0 & 0 & 0 & 0 & 5.565 \\
\hline & 344.35 & 70 & & 0.388 & 0.472 & 0.097 & 0.042 & 0.001 & 0 & 0 & 0 & 0 & 5.071 \\
\hline & 344.35 & 86 & & 0.371 & 0.459 & 0.125 & 0.043 & 0.001 & 0 & 0 & 0 & 0 & 4.646 \\
\hline & 344.35 & 110 & & 0.356 & 0.442 & 0.155 & 0.045 & 0.001 & 0 & 0 & 0 & 0 & 4.075 \\
\hline & 344.35 & 127 & & 0.326 & 0.431 & 0.193 & 0.048 & 0.001 & 0 & 0 & 0 & 0 & 3.714 \\
\hline & 344.35 & 143 & & 0.320 & 0.422 & 0.208 & 0.047 & 0.002 & 0 & 0 & 0 & 0 & 3.403 \\
\hline & 344.35 & 159 & & 0.291 & 0.414 & 0.240 & 0.053 & 0.001 & 0 & 0 & 0 & 0 & 3.118 \\
\hline & 344.35 & 176 & & 0.278 & 0.403 & 0.262 & 0.054 & 0.002 & 0 & 0 & 0 & 0 & 2.841 \\
\hline & 344.35 & 194 & & 0.265 & 0.393 & 0.285 & 0.055 & 0.002 & 0 & 0 & 0 & 0 & 2.575 \\
\hline & 344.35 & 211 & & 0.261 & 0.389 & 0.293 & 0.054 & 0.002 & 0 & 0 & 0 & 0 & 2.346 \\
\hline & 344.35 & 229 & & 0.236 & 0.384 & 0.318 & 0.060 & 0.002 & 0 & 0 & 0 & 0 & 2.127 \\
\hline & 344.35 & 251 & & 0.232 & 0.375 & 0.332 & 0.058 & 0.003 & 0.001 & 0 & 0 & 0 & 1.886 \\
\hline & 344.35 & 268 & & 0.218 & 0.372 & 0.346 & 0.060 & 0.003 & 0.001 & 0 & 0 & 0 & 1.718 \\
\hline & 344.35 & 285 & & 0.213 & 0.369 & 0.354 & 0.061 & 0.003 & 0.001 & 0 & 0 & 0 & 1.566 \\
\hline & 344.35 & 303 & & 0.195 & 0.368 & 0.374 & 0.059 & 0.003 & 0.001 & 0 & 0 & 0 & 1.419 \\
\hline & 344.35 & 318 & & 0.193 & 0.361 & 0.379 & 0.062 & 0.004 & 0.001 & 0 & 0 & 0 & 1.308 \\
\hline \multirow{13}{*}{$\begin{array}{l}\text { Batch } \\
\text { reactor }\end{array}$} & 355.55 & 0 & \multirow[t]{13}{*}{0.1238} & 0.503 & 0.497 & 0 & 0 & 0 & 0 & 0 & 0 & 0 & 17.464 \\
\hline & 355.55 & 2 & & 0.495 & 0.491 & 0.014 & 0.001 & 0 & 0 & 0 & 0 & 0 & 16.042 \\
\hline & 355.55 & 33 & & 0.259 & 0.309 & 0.422 & 0.002 & 0.006 & 0.002 & 0 & 0 & 0 & 4.300 \\
\hline & 355.55 & 51 & & 0.181 & 0.249 & 0.558 & 0.002 & 0.007 & 0.002 & 0 & 0 & 0 & 2.002 \\
\hline & 355.55 & 67 & & 0.154 & 0.224 & 0.609 & 0.002 & 0.008 & 0.002 & 0 & 0 & 0 & 1.015 \\
\hline & 355.55 & 83 & & 0.134 & 0.213 & 0.641 & 0.002 & 0.008 & 0.002 & 0 & 0 & 0 & 0.514 \\
\hline & 355.55 & 101 & & 0.133 & 0.209 & 0.645 & 0.002 & 0.008 & 0.002 & 0 & 0 & 0 & 0.239 \\
\hline & 355.55 & 118 & & 0.127 & 0.207 & 0.652 & 0.002 & 0.009 & 0.002 & 0 & 0 & 0 & 0.116 \\
\hline & 355.55 & 134 & & 0.122 & 0.207 & 0.657 & 0.002 & 0.009 & 0.003 & 0 & 0 & 0 & 0.059 \\
\hline & 355.55 & 154 & & 0.128 & 0.208 & 0.650 & 0.002 & 0.009 & 0.002 & 0 & 0 & 0 & 0.025 \\
\hline & 355.55 & 175 & & 0.130 & 0.212 & 0.645 & 0.002 & 0.009 & 0.002 & 0 & 0 & 0 & 0.010 \\
\hline & 355.55 & 194 & & 0.126 & 0.210 & 0.649 & 0.002 & 0.010 & 0.003 & 0 & 0 & 0 & 0.005 \\
\hline & 355.55 & 232 & & 0.124 & 0.213 & 0.648 & 0.002 & 0.010 & 0.003 & 0 & 0 & 0 & 0.001 \\
\hline \multirow{14}{*}{$\begin{array}{l}\text { Batch } \\
\text { reactor }\end{array}$} & 334.25 & 0 & \multirow[t]{14}{*}{0.3530} & 0.500 & 0.500 & 0 & 0 & 0 & 0 & 0 & 0 & 0 & 3.396 \\
\hline & 334.25 & 3 & & 0.492 & 0.496 & 0.012 & 0 & 0 & 0 & 0 & 0 & 0 & 3.206 \\
\hline & 334.25 & 34 & & 0.353 & 0.372 & 0.266 & 0.001 & 0.007 & 0.001 & 0 & 0 & 0 & 1.773 \\
\hline & 334.25 & 65 & & 0.249 & 0.298 & 0.439 & 0.002 & 0.010 & 0.002 & 0 & 0 & 0 & 0.980 \\
\hline & 334.25 & 98 & & 0.181 & 0.235 & 0.568 & 0.002 & 0.011 & 0.003 & 0 & 0 & 0 & 0.522 \\
\hline & 334.25 & 128 & & 0.135 & 0.194 & 0.654 & 0.002 & 0.012 & 0.003 & 0 & 0 & 0 & 0.294 \\
\hline & 334.25 & 165 & & 0.139 & 0.143 & 0.710 & 0.000 & 0.007 & 0.000 & 0 & 0 & 0 & 0.145 \\
\hline & 334.25 & 197 & & 0.114 & 0.133 & 0.745 & 0.000 & 0.007 & 0.001 & 0 & 0 & 0 & 0.079 \\
\hline & 334.25 & 229 & & 0.085 & 0.148 & 0.749 & 0.002 & 0.014 & 0.003 & 0 & 0 & 0 & 0.043 \\
\hline & 334.25 & 286 & & 0.078 & 0.143 & 0.760 & 0.002 & 0.014 & 0.003 & 0 & 0 & 0 & 0.014 \\
\hline & 334.25 & 365 & & 0.079 & 0.139 & 0.763 & 0.002 & 0.014 & 0.003 & 0 & 0 & 0 & 0.003 \\
\hline & 334.25 & 413 & & 0.075 & 0.140 & 0.766 & 0.002 & 0.014 & 0.003 & 0 & 0 & 0 & 0.001 \\
\hline & 334.25 & 440 & & 0.077 & 0.140 & 0.764 & 0.002 & 0.014 & 0.003 & 0 & 0 & 0 & 0.001 \\
\hline & 334.25 & 470 & & 0.079 & 0.140 & 0.762 & 0.002 & 0.014 & 0.003 & 0 & 0 & 0 & 0.000 \\
\hline \multirow{6}{*}{$\begin{array}{l}\text { Batch } \\
\text { reactor }\end{array}$} & 333.15 & 0 & \multirow[t]{6}{*}{0.2060} & 0.501 & 0.499 & 0 & 0 & 0 & 0 & 0 & 0 & 0 & 3.347 \\
\hline & 333.15 & 1 & & 0.500 & 0.499 & 0.001 & 0 & 0 & 0 & 0 & 0 & 0 & 3.306 \\
\hline & 333.15 & 33 & & 0.404 & 0.431 & 0.146 & 0.001 & 0.015 & 0.003 & 0 & 0 & 0 & 2.215 \\
\hline & 333.15 & 67 & & 0.303 & 0.372 & 0.290 & 0.001 & 0.027 & 0.007 & 0 & 0 & 0 & 1.447 \\
\hline & 333.15 & 99 & & 0.244 & 0.325 & 0.386 & 0.001 & 0.035 & 0.009 & 0 & 0 & 0 & 0.970 \\
\hline & 333.15 & 131 & & 0.198 & 0.289 & 0.464 & 0.001 & 0.039 & 0.010 & 0 & 0 & 0 & 0.650 \\
\hline
\end{tabular}




\begin{tabular}{|c|c|c|c|c|c|c|c|c|c|c|c|c|c|}
\hline \multirow{7}{*}{$\begin{array}{l}\text { Exper. } \\
\text { setup }\end{array}$} & \multirow{2}{*}{$\begin{array}{c}T \\
(\mathrm{~K})\end{array}$} & \multirow{2}{*}{$\begin{array}{c}t \\
(\min )\end{array}$} & \multirow{7}{*}{$\begin{array}{c}W_{\text {cat }} \\
\text { (g) }\end{array}$} & \multicolumn{9}{|c|}{ Mole fraction } & \multirow{2}{*}{$\begin{array}{c}r_{\mathrm{BTBE}} \\
{[\mathrm{mol} / \mathrm{g} \mathrm{h}]}\end{array}$} \\
\hline & & & & $x_{1 \mathrm{~B}}$ & $\boldsymbol{X}_{\mathrm{BuOH}}$ & $\boldsymbol{X}_{\mathrm{BTBE}}$ & $\boldsymbol{x}_{\text {TBA }}$ & $\boldsymbol{X}_{\text {TMP-1 }}$ & $x_{\text {TMP-2 }}$ & $\boldsymbol{X}_{i \text {-butane }}$ & $\begin{array}{c}\boldsymbol{x}_{\text {trans-2- }} \\
\text { butene }\end{array}$ & $\begin{array}{l}\boldsymbol{X}_{\text {cis-2- }} \\
\text { butene }\end{array}$ & \\
\hline & 333.15 & 163 & & 0.164 & 0.261 & 0.523 & 0.001 & 0.042 & 0.010 & 0 & 0 & 0 & 0.435 \\
\hline & 333.15 & 199 & & 0.142 & 0.236 & 0.565 & 0.001 & 0.044 & 0.011 & 0 & 0 & 0 & 0.278 \\
\hline & 333.15 & 231 & & 0.120 & 0.222 & 0.601 & 0.001 & 0.046 & 0.011 & 0 & 0 & 0 & 0.186 \\
\hline & 333.15 & 275 & & 0.105 & 0.208 & 0.627 & 0.001 & 0.048 & 0.011 & 0 & 0 & 0 & 0.107 \\
\hline & 333.15 & 309 & & 0.091 & 0.199 & 0.651 & 0.002 & 0.046 & 0.012 & 0 & 0 & 0 & 0.070 \\
\hline \multirow{10}{*}{$\begin{array}{l}\text { Batch } \\
\text { reactor }\end{array}$} & 344.45 & 0 & \multirow[t]{10}{*}{0.1200} & 0.490 & 0.510 & 0 & 0 & 0 & 0 & 0 & 0 & 0 & 7.125 \\
\hline & 344.45 & 2 & & 0.488 & 0.506 & 0.006 & 0 & 0 & 0 & 0 & 0 & 0 & 6.895 \\
\hline & 344.45 & 36 & & 0.341 & 0.444 & 0.183 & 0.002 & 0.024 & 0.006 & 0 & 0 & 0 & 3.946 \\
\hline & 344.45 & 56 & & 0.285 & 0.391 & 0.282 & 0.002 & 0.032 & 0.008 & 0 & 0 & 0 & 2.841 \\
\hline & 344.45 & 91 & & 0.185 & 0.324 & 0.441 & 0.002 & 0.038 & 0.010 & 0 & 0 & 0 & 1.599 \\
\hline & 344.45 & 109 & & 0.177 & 0.282 & 0.485 & 0.002 & 0.044 & 0.011 & 0 & 0 & 0 & 1.190 \\
\hline & 344.45 & 130 & & 0.151 & 0.260 & 0.530 & 0.002 & 0.046 & 0.012 & 0 & 0 & 0 & 0.843 \\
\hline & 344.45 & 146 & & 0.129 & 0.252 & 0.559 & 0.002 & 0.046 & 0.012 & 0 & 0 & 0 & 0.648 \\
\hline & 344.45 & 163 & & 0.115 & 0.242 & 0.579 & 0.002 & 0.049 & 0.013 & 0 & 0 & 0 & 0.490 \\
\hline & 344.45 & 180 & & 0.121 & 0.232 & 0.590 & 0.002 & 0.043 & 0.012 & 0 & 0 & 0 & 0.371 \\
\hline \multirow{14}{*}{$\begin{array}{l}\text { Batch } \\
\text { reactor }\end{array}$} & 343.45 & 0 & \multirow[t]{14}{*}{0.1520} & 0.455 & 0.545 & 0 & 0 & 0 & 0 & 0 & 0 & 0 & 4.974 \\
\hline & 343.45 & 5 & & 0.399 & 0.528 & 0.030 & 0.042 & 0 & 0 & 0 & 0 & 0 & 4.643 \\
\hline & 343.45 & 42 & & 0.316 & 0.436 & 0.243 & 0.001 & 0.002 & 0.001 & 0 & 0 & 0 & 2.790 \\
\hline & 343.45 & 73 & & 0.234 & 0.386 & 0.374 & 0.002 & 0.003 & 0.001 & 0 & 0 & 0 & 1.821 \\
\hline & 343.45 & 124 & & 0.186 & 0.337 & 0.470 & 0.002 & 0.004 & 0.001 & 0 & 0 & 0 & 0.903 \\
\hline & 343.45 & 156 & & 0.141 & 0.311 & 0.541 & 0.002 & 0.004 & 0.001 & 0 & 0 & 0 & 0.581 \\
\hline & 343.45 & 197 & & 0.122 & 0.291 & 0.579 & 0.002 & 0.005 & 0.001 & 0 & 0 & 0 & 0.331 \\
\hline & 343.45 & 232 & & 0.092 & 0.281 & 0.619 & 0.002 & 0.005 & 0.001 & 0 & 0 & 0 & 0.204 \\
\hline & 343.45 & 273 & & 0.092 & 0.274 & 0.626 & 0.002 & 0.005 & 0.001 & 0 & 0 & 0 & 0.116 \\
\hline & 343.45 & 304 & & 0.084 & 0.270 & 0.637 & 0.002 & 0.005 & 0.001 & 0 & 0 & 0 & 0.076 \\
\hline & 343.45 & 341 & & 0.079 & 0.267 & 0.646 & 0.002 & 0.005 & 0.001 & 0 & 0 & 0 & 0.046 \\
\hline & 343.45 & 372 & & 0.075 & 0.266 & 0.651 & 0.002 & 0.006 & 0.001 & 0 & 0 & 0 & 0.030 \\
\hline & 343.45 & 406 & & 0.073 & 0.265 & 0.654 & 0.002 & 0.006 & 0.001 & 0 & 0 & 0 & 0.019 \\
\hline & 343.45 & 435 & & 0.073 & 0.265 & 0.654 & 0.002 & 0.006 & 0.001 & 0 & 0 & 0 & 0.013 \\
\hline
\end{tabular}




\section{Sensitivity analysis of the parameters on the selected kinetic model}

The sensitivity analysis of the parameters on the reaction rate predicted by the selected model (Model 49, Equation 18) has been evaluated by means of the Monte Carlo method. It accounts for the effect of the parameter uncertainty on the model output. The applied procedure consists of 3 steps:

1) Random generation of a set of parameters: Parameters in the model were assumed to follow a normal distribution with mean equal to its optimal value and standard deviation equal to its standard error, both estimated from the fit. For each parameter, a random value was generated according to its normal distribution. As a result, a set of random parameters was obtained and it was used to calculate the reaction rates at the conditions of each experimental point using Equation 18.

2) For each experimental point, the previous step was repeated 1000 times to obtain 1000 calculated reaction rates values. They followed a normal distribution. Its mean corresponds to the reaction rate predicted with Equation 18 using the optimal parameter values. Its standard deviation is a measure of the model sensitivity due to the uncertainty of the parameters.

3) Error bars in Figure 4(a) show the model output sensitivity, expressed as the standard deviation of the calculated reaction rates given the standard error of the fitted parameters. 


\section{Best models ranking obtained with the fixed resin solubility parameter value}

TABLE S2. Optimal parameter values for BTBE kinetic equations with the restriction that $\delta_{\mathrm{P}}=20.85 \mathrm{MPa}^{1 / 2}$, when $\delta_{\mathrm{P}}$ is included. A "-" sign indicates that the related effect is not included in the model.

\begin{tabular}{|c|c|c|c|c|c|c|c|c|c|c|c|c|c|c|c|}
\hline \multirow{2}{*}{ Model\# } & \multicolumn{2}{|c|}{$k^{\prime}(\mathrm{mol} / \mathrm{g} \mathrm{h})$} & \multirow{2}{*}{$\begin{array}{l}\text { \{driving } \\
\text { force }^{\text {a }}\end{array}$} & \multicolumn{7}{|c|}{ \{adsorption term\} } & \multirow[b]{2}{*}{$n$} & \multirow{2}{*}{$\begin{array}{l}\delta_{\mathrm{p}} \quad \text { included } \\
\text { (Equation 9) }\end{array}$} & \multirow{2}{*}{ RSS } & \multirow{2}{*}{$\Delta_{i}$} & \multirow{2}{*}{$w_{i}$} \\
\hline & $k_{1}^{\prime}$ & $k_{T}^{\prime}$ & & $1^{\text {st }} \mathrm{Ads}^{\mathrm{b}}$ & $K_{1, \mathrm{BuOH}}$ & $K_{\mathrm{T}, \mathrm{BuOH}}$ & $K_{1, \mathrm{IB}}$ & $K_{\mathrm{T}, \mathrm{BB}}$ & $K_{1, \mathrm{BTBE}}$ & $K_{\mathrm{T}, \mathrm{BTBE}}$ & & & & & \\
\hline 49 & 0.686 & -9570 & (a) & $a_{\mathrm{BuOH}}$ & - & - & - & - & -1.890 & -4991 & 1 & Yes & 7.364 & 0 & 0.447 \\
\hline 151 & 0.231 & -9491 & (a) & $a_{\mathrm{BuOH}}$ & - & - & - & - & -1.862 & -3199 & 2 & Yes & 7.541 & 3 & 0.089 \\
\hline 48 & 0.709 & -9440 & (a) & $a_{\mathrm{BuOH}}$ & - & - & - & - & -1.096 & - & 1 & Yes & 7.687 & 4 & 0.071 \\
\hline 736 & 0.233 & -9487 & (c) & $a_{\mathrm{BuOH}}$ & - & - & - & - & -1.150 & -3711 & 1 & Yes & 7.581 & 4 & 0.062 \\
\hline 156 & 3.641 & -9396 & (a) & 1 & 1.350 & - & - & - & 0.103 & - & 2 & Yes & 7.606 & 4 & 0.050 \\
\hline 273 & 3.121 & -9393 & (a) & 1 & 0.370 & - & - & - & -0.884 & - & 3 & Yes & 7.616 & 5 & 0.046 \\
\hline 964 & 0.712 & -9429 & (c) & 1 & - & - & - & - & -1.673 & - & 3 & Yes & 7.786 & 5 & 0.030 \\
\hline 847 & 0.712 & -9426 & (c) & 1 & - & - & - & - & -1.248 & - & 2 & Yes & 7.787 & 5 & 0.030 \\
\hline 730 & 0.714 & -9420 & (c) & 1 & - & - & - & - & -0.497 & - & 1 & Yes & 7.792 & 6 & 0.028 \\
\hline 157 & 3.941 & -8540 & (a) & 1 & 1.559 & 597.6 & - & - & 0.209 & - & 2 & Yes & 7.563 & 6 & 0.024 \\
\hline 741 & 1.237 & -9380 & (c) & 1 & 0.075 & - & - & - & 0.198 & - & 1 & Yes & 7.701 & 6 & 0.021 \\
\hline 858 & 1.124 & -9388 & (c) & 1 & -1.028 & - & - & - & -0.873 & - & 2 & Yes & 7.711 & 6 & 0.020 \\
\hline 975 & 1.093 & -9391 & (c) & 1 & -1.549 & - & - & - & -1.378 & - & 3 & Yes & 7.715 & 6 & 0.019 \\
\hline 274 & 3.199 & -9004 & (a) & 1 & 0.425 & 277.0 & - & - & -0.874 & - & 3 & Yes & 7.599 & 6 & 0.018 \\
\hline 150 & 0.256 & -9354 & (a) & $a_{\mathrm{BuOH}}$ & - & - & - & - & -1.355 & - & 2 & Yes & 7.895 & 7 & 0.012 \\
\hline 742 & 15.10 & -6558 & (c) & 1 & 14.88 & 3040 & - & - & 13.828 & - & 1 & Yes & 7.654 & 7 & 0.011 \\
\hline 859 & 1.158 & -9287 & (c) & 1 & -0.935 & 270.7 & - & - & -0.857 & - & 2 & Yes & 7.705 & 8 & 0.007 \\
\hline 976 & 1.116 & -9314 & (c) & 1 & -1.485 & 225.7 & - & - & -1.371 & - & 3 & Yes & 7.711 & 8 & 0.007 \\
\hline 735 & 0.260 & -9339 & (c) & $a_{\mathrm{BuOH}}$ & - & - & - & - & -0.568 & - & 1 & Yes & 7.974 & 9 & 0.006 \\
\hline 56 & 707.6 & -9696 & (a) & 1 & 706.9 & - & - & - & 705.8 & - & 1 & Yes & 7.887 & 9 & 0.004 \\
\hline 2 & 1.116 & -9694 & (a) & 1 & - & - & - & - & - & - & 1 & Yes & 9.692 & 33 & $<10^{-6}$ \\
\hline
\end{tabular}

${ }^{a}$ Form of the driving force: (a) surface reaction (Equation 3 ) and (c) isobutene adsorption (Equation 5 ). ${ }^{b}$ First summand of the adsorption term. 